\title{
Misdiagnosis of Li-Fraumeni Syndrome in a Patient With Clonal Hematopoiesis and a Somatic TP53 Mutation
}

\author{
Rachel L. Mitchell, MD; ; Cory Kosche, BSª Kelly Burgess, MS, CGC; ; Shreya Wadhwa, BSa;

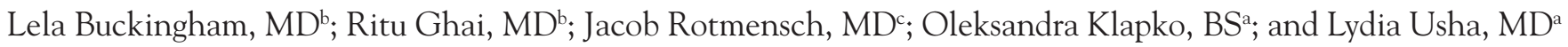

\begin{abstract}
Li-Fraumeni syndrome (LFS) is a rare genetic disorder that confers a high risk of developing certain malignancies at a young age. It is caused by germline mutations in the TP53 gene and is typically diagnosed by sequencing this gene in blood cells. The presence of a mutation in approximately half of the DNA reads (allelic fraction of 50\%) is an indicator of a germline mutation, such as that in LFS. Clonal hematopoiesis $(\mathrm{CH})$ is an expansion of a hematopoietic clone containing a somatic driver mutation with a low allelic fraction, usually not more than $10 \%$ to $20 \%$. This report presents a patient with fallopian tube carcinoma who underwent multigene panel testing for cancer predisposition and was found to have a mutation in the TP53 gene, c.733G>T (p.Gly245Cys). Since the TP53 mutation had an allelic fraction of approximately $50 \%$, it was interpreted as being germline, and the patient was diagnosed as having LFS. A year later, she developed acute myelogenous leukemia. Subsequent mutational analysis showed that the TP53 mutation was absent in her benign tissue sample but present in leukemic cells. Furthermore, sequencing of the fallopian tube tumor tissue revealed a different TP53 gene mutation, c.818G>T (p.Arg273Leu). These observations confirmed that the previously identified mutation in her blood was somatic rather than germline and that she had $\mathrm{CH}$ at the time of genetic testing. $\mathrm{CH}$ can occasionally lead to a misdiagnosis of a germline mutation and a cancer predisposition syndrome that has significant implications for patients and their families. Therefore, the abnormal result of genetic testing for hereditary cancer susceptibility should be carefully interpreted when the clinical presentation is atypical, when the patient is older, when the gene in question is known to have potential germline and somatic mutations such as the TP53 gene, and when the allelic fraction is approximately $50 \%$.
\end{abstract}

J Natl Compr Canc Netw 2018;16(5):461-466 doi: 10.6004/jnccn.2017.7058

Li-Fraumeni syndrome (LFS) is a rare, autosomal dominant disorder that confers a high risk of developing cancer, such as sarcomas and breast, brain, and adrenocortical cancers. In patients with LFS, these cancers are generally diagnosed before 30 years of age. The estimated carrier rate frequency is between 1:5,000 and 1:20,000 in the general population. LFS is caused by alterations in the tumor suppressor gene TP53, with $>300$ different germline mutations identified thus far. ${ }^{1}$ Some patients do not meet classic LFS diagnostic criteria but display similar, often milder, phenotypes and are classified as having Li-Fraumeni-like syndrome (LFLS). Most families with LFLS do not harbor detectable germline TP53 mutations

aDepartment of Hematology, Oncology, and Stem Cell Transplant Medicine; 'Department of Pathology; and 'Division of Gynecologic Oncology, Department of Obstetrics and Gynecology, Rush University Medical Center, Chicago, Illinois.

Submitted July 6, 2017; accepted for publication November 12, 2017. in the coding region of the gene. ${ }^{2}$ This report presents a misdiagnosis of LFS in a patient with fallopian tube carcinoma. On further analysis, a TP53 mutation identified in her blood sample was determined to be a somatic mutation present in a hematopoietic clone consistent with clonal hematopoiesis $(\mathrm{CH})$, which subsequently transformed into acute myelogenous leukemia (AML).

$\mathrm{CH}$ is a form of somatic mosaicism with the clonal expansion of a single mutant hematopoietic cell line and is found in approximately $10 \%$ of persons aged $>65$ years. ${ }^{3}$ It can represent a premalignant state associated with potential progression to a myelodysplastic syndrome and further to AML. CH is caused by the ac-
The authors have disclosed that they have no financial interests, arrangements, affiliations, or commercial interests with the manufacturers of any products discussed in this article or their competitors.

Correspondence: Cory Kosche, BS, 644 West Roscoe Street \#2, Chicago, IL 60657. Email: Cory_A_Kosche@rush.edu 
Mitchell et al

quisition of a driver mutation in the hematopoietic cell line. ${ }^{4}$ It is usually detected when the mutation is present with an allelic fraction approximately $10 \%$ to $\leq 20 \%$ (the mutation is present in $10 \%-20 \%$ of analyzed DNA reads) (Figure 1). However, because the driver mutation confers a proliferative advantage to the clone, its allelic fraction can increase to $\geq 50 \%$. The most commonly mutated genes include DNMT3A, TET2, ASXL1, PPM1D, and TP53. Subclonal lines can then develop with additional mutations that lead to the development of a hematologic malignancy, with an absolute risk of conversion from $\mathrm{CH}$ to malignancy of approximately $1.0 \%$ per year. In one study, clonal lines were identified as cells that had a significantly elevated allelic fraction of a mutant gene that remained $<50 \%$. Mutant genes with an allelic fraction of approximately $50 \%$ were classified as inherited (germline) mutations. ${ }^{3}$

\section{Case Report}

The patient was diagnosed with stage IIIC fallopian tube carcinoma in June 2005 at age 69 years. She underwent a total abdominal hysterectomy with bilateral oophorectomy and surgical debulking. Pathology was consistent with a high-grade serous carcinoma. Immunohistochemical staining was positive for TP53 (Figure 2), WT-1, and ER. She received adjuvant chemotherapy with 6 cycles of carboplatin and paclitaxel. She remained disease-free for 44 months, when she developed a nodal recurrence and was re-treated with carboplatin and paclitaxel. She subsequently received multiple lines of therapy after her initial relapse, including gemcitabine, liposomal doxorubicin, singleagent carboplatin, tamoxifen, and anastrozole.

Because the patient had a personal history of fallopian tube carcinoma, she was a candidate for genetic testing for BRCA1 and BRCA2 mutations. Additionally, due to a family history significant for multiple malignancies on both sides of her family (Figure 3), she was referred to a genetic counselor. With the development of next-generation sequencing, gene panels are increasingly being used as potentially the more efficient and cost-effective methods of genetic testing. In June 2014, the OncoGeneDx Breast/Ovarian Cancer Panel (GeneDx) was performed on her blood sample to evaluate the following genes: ATM, BARD1, BRCA1, BRCA2, BRIP1, CDH1, CHEK2, EPCAM, FANCC, MLH1, MSH2, MSH6, NBN, PALB2, PMS2, PTEN, RAD51C, STK11, TP53, and XRCC2. She was found to have a deleterious mutation in the TP53 gene, c.733G > T (p.Gly245Cys). Clinically, she met the Eeles criteria for LFLS, which requires the presence of 2 first- or second-degree relatives with LFS-associated malignancies. ${ }^{5}$ In her case, she had a first-degree relative with breast cancer (mother) and a second-degree relative with a brain tumor (nephew). Based on the presence of the presumed germline mutation, LFS was diagnosed and genetic testing for her mutation

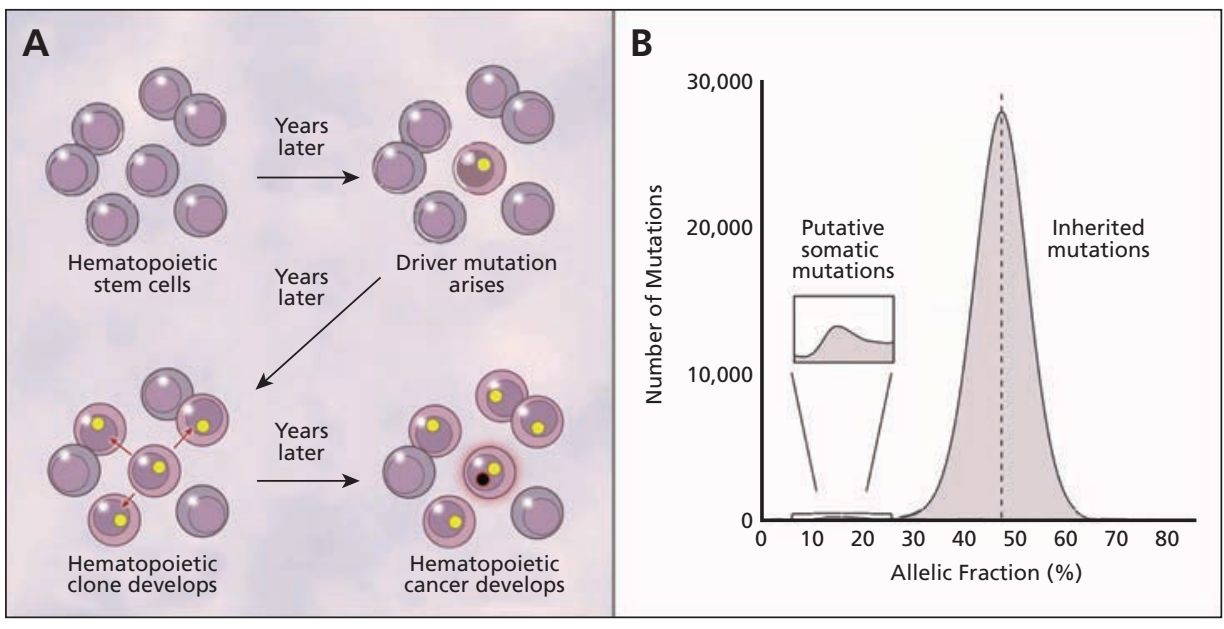

Figure 1. (A) The model for clonal hematopoiesis $(\mathrm{CH})$ and its influence by a driver mutation followed by the development of a hematologic cancer on acquisition of an additional mutation. (B) $\mathrm{CH}$ is defined by the expansion of a single cell line to an extent where a single mutation becomes present with an elevated allelic fraction. Notably, the authors chose to classify mutations with an allelic fraction near $50 \%$ as inherited mutations.

From The New England Journal of Medicine, Genovese G, Kahler AK, Handsaker RE, et al, Clonal hematopoiesis and blood-cancer risk inferred from blood DNA sequence, 371:2479. Copyright @ 2014 Massachusetts Medical Society. Reprinted with permission from Massachusetts Medical Society. 


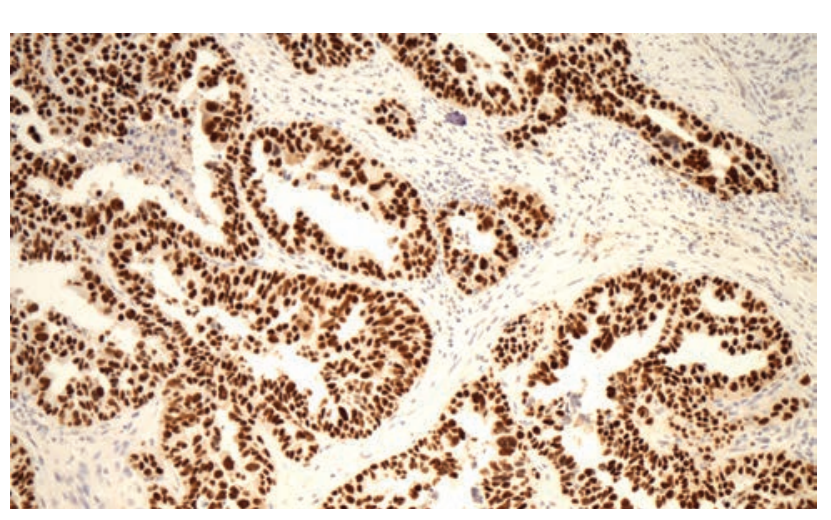

Figure 2. Positive immunohistochemical staining for mutant TP53 protein with DAKO DO-7 stain on a pathology slide from the patient's fallopian tube carcinoma (original magnification x10).

was recommended to family members. To our knowledge, none of them underwent genetic testing.

In 2015, the patient developed therapy-related AML with characteristic del5q and del7q chromosomal abnormalities. The patient ultimately passed away from AML in September 2015.

As mentioned, our patient had fallopian tube carcinoma, which is not a cancer commonly associated with LFS or LFLS. Furthermore, she was diagnosed with this cancer at age 69 years, which is not a typical LFS presentation. In addition, there was no compelling family history for LFS. After the patient died of therapy-related AML, we began to question the diagnosis of LFS and sought to revisit it. We obtained a benign tissue sample from a previous laparotomy for an unrelated ileocolic anastomosis in 2013.
For further analysis, we retrieved a 2015 sample of the leukemic bone marrow and performed TP53 mutational analysis on both specimens. The samples were tested, first by single-strand conformation polymorphism, wherein a band shift was detected in exon 7 of the bone marrow but not detected in the benign tissue. DNA from exon 7 of both specimens was then subjected to Sanger sequencing, revealing the TP53 c.733G > T (p.Gly245Cys) mutation in the leukemic bone marrow but not in the benign tissue (Figure 4). If the mutation were germline, it should have been ubiquitously present in all tissues.

To further determine if the mutation found in the blood/bone marrow was a somatic TP53 mutation, tumor DNA from the fallopian tube carcinoma was analyzed using a gene panel (TruSight Tumor 15, Illumina, Inc.) that included AKT1, BRAF, EGFR, ERBB2, FOXL1, GNA11, GNAQ, KIT, KRAS, MET, NRAS, PDGFRA, PIK3CA, RET, and TP53 genes. This analysis revealed a c.818G $>$ T (p.Arg273Leu) mutation in the TP53 gene, different from the mutation detected in blood and bone marrow.

The lack of an identical mutation in the benign tissue and fallopian tumor tissue indicated that the patient's mutation was somatic, not germline, and that the diagnosis of LFS was incorrect.

\section{Discussion}

LFS is caused by germline mutations in TP53, a tumor suppressor gene located on chromosome 17. In-

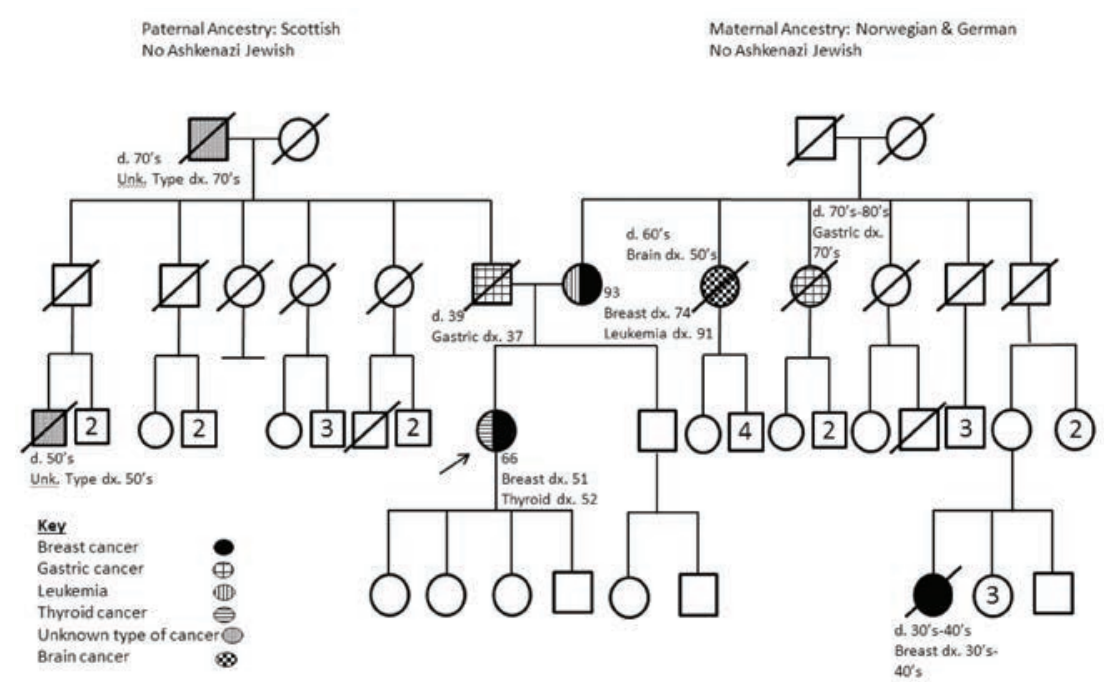

Figure 3. The patient's pedigree (proband, indicated by arrow).

Abbreviations: $d$, age at death; dx, age at diagnosis; unk, unknown. 
Tissue CGC

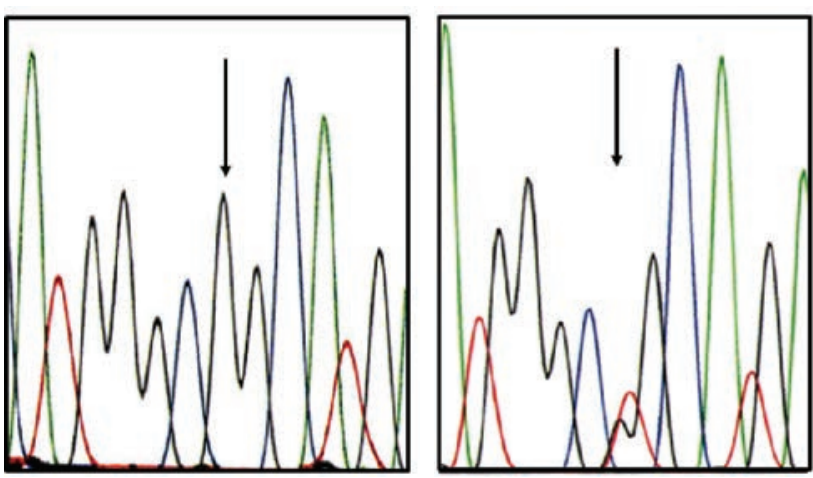

Figure 4. Sanger sequencing results from benign abdominal tissue (left) and bone marrow (right). A ' $\mathrm{T}$ ' (red) nucleotide peak was detected at base 733 in exon 7 of the bone marrow but not detected in the benign tissue, revealing the TP53 c.733G>T (p.Gly245Cys) mutation in the leukemic bone marrow but not in the benign tissue.

dividuals with LFS have a 50\% risk of developing cancer before age 30 years compared with the estimated $1 \%$ risk in the general population. ${ }^{6}$ LFS is defined as "a proband with a sarcoma diagnosed before age 45 years; a first-degree relative with any cancer before age 45; and a first- or second-degree relative with any cancer before age 45 or sarcoma at any age." 7 Further research has shown that many other malignancies can be manifestations of LFS, such as gastric cancer, lymphoma, melanoma, choroid plexus carcinoma, colorectal cancer, germ cell tumor, and Wilms tumor, ${ }^{8}$ but that LFS is infrequently associated with fallopian tube/ovarian cancer. ${ }^{9}$ Families that do not meet criteria for classic LFS are considered to have LFLS. These families were initially classified based on either the Birch or Eeles criteria. More recently, the revised Chompret criteria were proposed to include classic LFS and some families with LFLS. The criteria include the following personal and family history: (1) a proband with a characteristic LFS tumor before age 46 years who has at least one first- or second-degree relative with a characteristic LFS tumor before age 46 years; (2) a proband with multiple tumors, 2 of which represent characteristic LFS tumors and the first of which occurred before age 46 years; or (3) a proband with adrenocortical carcinoma irrespective of age or family history. ${ }^{10}$ The Eeles criteria (2 first- or second-degree relatives with LFS-associated malignancies) are the least stringent, and therefore have the lowest specificity (16\%) for detecting germline TP53 mutations. ${ }^{11}$
Germline TP53 mutations have been detected in $56 \%$ of families meeting the classic LFS criteria and in 35\% of families meeting the Chompret criteria. ${ }^{11}$ As mentioned, most LFLS families do not harbor germline TP53 mutations. ${ }^{2,12,13}$ In fact, they have been detected in only $14 \%$ of families meeting the Eeles criteria. ${ }^{11}$

TP53 mutations lead to an abnormal p53 protein, which allows cells containing damaged DNA to survive and proliferate, contributing to malignant transformation. ${ }^{14}$ Somatic mutations and deletions in TP53 have also been observed in $>50 \%$ of sporadic human cancers. ${ }^{15}$ Somatic mutations in TP53 with positive immunostaining of the tumor are typical in fallopian tube carcinomas (as was in our case; Figure 2) and should not cause suspicion of a hereditary cancer syndrome. ${ }^{9}$

Most mutations in the TP53 gene are missense mutations and occur in the DNA-binding region of the gene, exons 5 through $8 .{ }^{1}$ In our patient, the mutation was located in codon 245 , which is one of the most common locations for both germline and somatic TP53 mutations, along with codons 175, 248, 273, and 282, all in the DNA-binding domain. ${ }^{8} \mathrm{Mu}$ tational analysis of the patient's blood cells revealed a p.G245C mutation in the TP53 gene, where the glycine at codon 245 is replaced by cysteine, resulting in an amino acid substitution in exon 6 of the DNA-binding domain. This mutation was initially described in a patient diagnosed with osteosarcoma at age 11 years who had 2 first-degree relatives that developed sarcomas at ages 19 and 58 years. ${ }^{14}$ Several studies have attempted to explain the pathogenesis of this mutation and one study categorized the mutation as a severe deficiency or nonfunctional allele because it is devoid of transactivation capacity. Another group showed that the p.G245C mutation led to greatly reduced DNA-binding ability compared with wild-type p53, likely because it results in a change in the conformation of the DNA-binding motif. ${ }^{16-18}$

Our patient was diagnosed with LFS by a reputable genetic testing laboratory based on the presence of the TP53 mutation, c.733G >T (p.Gly245Cys), with a "heterozygous" inheritance. With no additional personal history of cancer and a late presentation of fallopian tube carcinoma, this patient did not meet the classic or Chompret clinical criteria for the diagnosis of LFS. Failure to demonstrate c.733G >T (p.Gly245Cys) mutation in her archival benign tis- 


\begin{tabular}{|c|c|c|c|}
\hline & $\begin{array}{l}\text { Normal } \\
\text { Values }\end{array}$ & May 29, 2014 & July 29, 2014 \\
\hline WBC $\left(10^{3} / \mathrm{mcL}\right)$ & $4.2-11.0$ & 6.1 & 10.1 \\
\hline $\mathrm{RBC}\left(10^{6} / \mathrm{mcL}\right)$ & $4.00-5.20$ & 4.07 & 4.32 \\
\hline Hemoglobin (g/dL) & $12.0-15.5$ & 11.6 & 12.2 \\
\hline Hematocrit (\%) & $36.0-46.5$ & 34.8 & 36.4 \\
\hline $\mathrm{MCV}(\mathrm{fL})$ & $78.0-100.0$ & 85.5 & 84.3 \\
\hline $\mathrm{MCH}(p g)$ & $26.0-34.0$ & 28.5 & 28.2 \\
\hline $\mathrm{MCHC}(\mathrm{g} / \mathrm{dL})$ & $32.0-36.5$ & 33.3 & 33.5 \\
\hline RDW-CV (\%) & $11.0-15.0$ & 16.0 & 16.7 \\
\hline Platelets $\left(10^{3} / \mathrm{mcL}\right)$ & $140-450$ & 214 & 271 \\
\hline Segmental neutrophils & & N/A & N/A \\
\hline Neutrophils (\%) & & 48 & 70 \\
\hline Lymphocytes (\%) & & 41 & 16 \\
\hline Monocytes (\%) & & 8 & 6 \\
\hline Eosinophils (\%) & & 3 & 8 \\
\hline Basophils (\%) & & 0 & 0 \\
\hline $\begin{array}{l}\text { Absolute neutrophils } \\
\left(10^{3} / \mathrm{mcL}\right)\end{array}$ & $1.8-7.7$ & 2.9 & 7.1 \\
\hline $\begin{array}{l}\text { Absolute lymphocytes } \\
\left(10^{3} / \mathrm{mcL}\right)\end{array}$ & $1.0-4.0$ & 2.5 & 1.6 \\
\hline $\begin{array}{l}\text { Absolute monocytes } \\
\left(10^{3} / \mathrm{mcL}\right)\end{array}$ & $0.3-0.9$ & 0.5 & 0.6 \\
\hline $\begin{array}{l}\text { Absolute eosinophils } \\
\left(10^{3} / \mathrm{mcL}\right)\end{array}$ & $0.1-0.5$ & 0.2 & 0.8 \\
\hline $\begin{array}{l}\text { Absolute basophils } \\
\left(10^{3} / \mathrm{mcL}\right)\end{array}$ & $0.0-0.3$ & 0.0 & 0.0 \\
\hline
\end{tabular}

Insignificantly abnormal values are indicated in bold.

Abbreviations: $\mathrm{MCH}$, mean corpuscular hemoglobin; $\mathrm{MCHC}$, mean corpuscular hemoglobin concentration; MCV, mean corpuscular volume; N/A, not available; RBC, red blood cell count; RDW-CV, red blood cell distribution width; WBC, white blood cell count.

sue sample indicated that she did not have a germline TP53 mutation and that the mutation identified by the genetic testing laboratory was a somatic mutation present in her blood cells $(\mathrm{CH})$. The fact that she met the Eeles criteria for the diagnosis of LFLS made the interpretation of her genetic finding more difficult.

We suggest that in our patient, $\mathrm{CH}$ with an acquired somatic mutation in the TP53 gene, c.733G > T (p.Gly245Cys), had progressed to AML. At the time a blood sample was taken for genetic testing, the clonal cell line had achieved a state in which approximately $50 \%$ of cells contained this TP53 mutation. Her blood test results were unremarkable at that time (Table 1), as is commonly observed in $\mathrm{CH} .{ }^{4}$ Even though leukemia is often observed in LFS, our patient had been exposed to chemotherapy for ovarian cancer, and her leukemic cells had chromosomal abnormalities characteristic of treatment-induced leukemia.

$\mathrm{CH}$ is a form of somatic mosaicism, which is the development of a postzygotic de novo mutation resulting in 2 genotypically distinct cell lines in the same organism. Somatic mosaicism can occur at any time during ontogenesis, including embryonic and fetal periods, and after birth. The TP53 gene is one of the most commonly mutated genes in somatic mosaicism. If a TP53 mutation develops early in life, it may mimic LFS. ${ }^{19}$ This phenomenon is rare but has been reported in the literature, such as with the case of a young patient with early-onset adrenocortical adenoma and osteosarcoma. ${ }^{19}$ The DNA analysis of varied tissue samples from the same patient both containing the mutation and lacking it allows the diagnosis of somatic mosaicism to be established.

Somatic TP53 mutations have been commonly described in patients exposed to prior chemotherapy. It has been suggested that the mutation is not a direct result of the cytotoxic therapy, but rather that the clonal cell line carrying age-related TP53 mutations are resistant to chemotherapy and preferentially expand after treatment. ${ }^{20,21}$ Our patient's prior chemotherapy could have played a role in the expansion of the cell line and eventually the development of AML.

Another potential explanation for a TP53 mutation found in a blood sample can be somatic mosaicism due to a malignancy. In this case, the mutation is present on circulating tumor DNA isolated from the plasma. ${ }^{22}$ Somatic mosaic TP53 mutations have been observed in the blood of patients with solid tumors, especially women with ovarian carcinoma. ${ }^{20}$ This possibility should also be considered when interpreting a germline result, especially when the allelic fraction of a mutation is $<50 \%$. We have shown that the TP53 mutation identified in our patient's blood was different from the TP53 mutation found in her fallopian tube cancer.

Similar instances of misinterpreted germline results have been observed in the setting of cell-free fetal DNA (cffDNA) screening due to fetal-placental mosaicism. ${ }^{23}$ cffDNA screening is performed through taking a maternal blood sample and measuring the fetal DNA present in maternal circulation. cffDNA can be differentiated from maternal DNA based on its significantly smaller size. ${ }^{24} \mathrm{cffDNA}$ screenings are most commonly used to discover fetal chromosomal abnormalities. Most cffDNA in maternal circulation comes from the external layer of the placenta. ${ }^{25} \mathrm{cffD}$ NA has been shown to clear from maternal circulation within hours after birth, becoming undetectable. ${ }^{26}$ Because our patient was 78 years of age at the time of testing, fetal-placental mosaicism was not a consideration for her test result. However, it should 
Mitchell et al

be considered in women of childbearing age undergoing a germline genetic test when the result is discordant with the phenotypic presentation.

We believe this case reveals the potential for misdiagnosis of a cancer predisposition syndrome in the process of genetic testing. Such an erroneous diagnosis has serious implications for patients and their families. Testing laboratories are becoming aware of potential LFS misdiagnosis; one recently presented a small study in which one-third of all TP53 mutations identified by next-generation sequencing with allelic frequency of approximately 50\% were subsequently confirmed through skin fibroblast testing to be somatically acquired rather than germline. ${ }^{27}$

\section{Conclusions}

Multigene panels in genetic testing for cancer predisposition can sometimes yield results that are not expected based on a patient's personal and family history. Testing laboratories and ordering healthcare providers should carefully evaluate any abnormal re- sult in the clinical context. Occasionally, a somatic mutation in blood cells can be misinterpreted as a germline mutation. In $\mathrm{CH}$, a somatic mutation is usually present in a small fraction of blood cells. However, with clone expansion, the allelic fraction can increase and reach 50\% (the mutation is found in approximately half of the analyzed DNA) or more. The allelic fraction of approximately $50 \%$ can be mistakenly assumed to represent heterozygosity and a germline mutation. Clinicians should be aware of possible misdiagnosis with a cancer predisposition syndrome when the gene in question is known to have potential germline and somatic mutations, such as the TP53 gene, when the allelic fraction is approximately $50 \%$, when the patient is older, and when the clinical presentation is atypical for the suspected genetic syndrome. In most challenging cases, skin fibroblast testing can be used to differentiate somatic from germline pathogenic variants. Molecular tumor boards are an ideal forum to discuss the management of such challenging cases in a hospital or academic setting.

\section{References}

1. Li FP, Fraumeni JF Jr, Mulvihill JJ, et al. A cancer family syndrome in twentyfour kindreds. Cancer Res 1988;48:5358-5362.

2. Kleihues $P$, Schauble B, zur Hausen A, et al. Tumors associated with $\mathrm{p} 53$ germline mutations: a synopsis of 91 families. Am J Pathol 1997;150:1-13.

3. Genovese G, Kahler AK, Handsaker RE, et al. Clonal hematopoiesis and blood-cancer risk inferred from blood DNA sequence. N Engl J Med 2014:371:2477-2487.

4. Steensma D, Bejar R, Jaiswal S, et al. Clonal hematopoiesis of indeterminate potential and its distinction from myelodysplastic syndromes. Blood 2015;125:9-16.

5. Eeles RA. Germline mutations in the TP53 gene. Cancer Surv 1995;25:101124.

6. Sorrell AD, Espenschied CR, Culver JO, Weitzel JN. Tumor protein p53 (TP53) testing and Li-Fraumeni syndrome: current status of clinical applications and future directions. Mol Diagn Ther 2013;17:31-47.

7. International Agency for Research on Cancer. Refs corner: Li-Fraumeni syndrome. Available at: http://p53.iarc.fr/RefsLiFraumeni.aspx. Accessed May 3, 2018.

8. Malkin D. Li-Fraumeni syndrome. Genes Cancer 2011;2:475-484.

9. International Agency for Research on Cancer. IARC TP53 Database, R17, November 2013. Tumors Associated with TP53 Germline Mutations. Available at: p53.iarc.fr/TP53GermlineMutations.aspx. Accessed April 9, 2018.

10. Tinat J, Bougeard G, Baert-Desurmont S, et al. 2009 version of the Chompret criteria for Li Fraumeni syndrome. J Clin Oncol 2009;27:108-109.

11. Gonzalez KD, Noltner KA, Buzin CH, et al. Beyond Li Fraumeni syndrome: clinical characteristics of families with $\mathrm{p} 53$ germline mutations. J Clin Oncol 2009;27:1250-1256.

12. Isobe M, Emanuel BS, Givol D, et al. Localization of gene for human $\mathrm{p} 53$ tumour antigen to band 17p13. Nature 1986;320:84-85.

13. Varley JM, Attwooll C, White G, et al. Characterization of germline TP53 splicing mutations and their genetic and functional analysis. Oncogene 2001;20:2647-2654.

14. Merino D, Malkin D. P53 and hereditary cancer. Subcell Biochem 2014;85:1-16.

15. Baker SJ, Fearon ER, Nigro JM, et al. Chromosome 17 deletions and $\mathrm{p} 53$ gene mutations in colorectal carcinomas. Science 1989;244:217-221.

16. Monti P, Perfumo C, Bisio A, et al. Dominant-negative features of mutant TP53 in germline carriers have limited impact on cancer outcomes. Mol Cancer Res 2011;9:271-279.

17. Malcikova J, Tichy B, Damborsky J, et al. Analysis of the DNA-binding activity of 553 mutants using functional protein microarrays and its relationship to transcriptional activation. Biol Chem 2010;391:197-205.

18. Pintus SS, Ivanisenko NV, Demenkov PS, et al. The substitutions G245C and G245D in the $\mathrm{Zn}(2+)$-binding pocket of the p53 protein result in differences of conformational flexibility of the DNA-binding domain. J Biomol Struct Dyn 2013;31:78-86.

19. Prochazkova K, Pavlikova K, Minarik M, et al. Somatic TP53 mutation mosaicism in a patient with Li-Fraumeni syndrome. Am J Med Genet 2009;149:206-211.

20. Swisher EM, Harrell MI, Norquist BM, et al. Somatic mosaic mutations in PPM1D and TP53 in the blood of women with ovarian carcinoma. JAMA Oncol 2016;2:370-372.

21. Wong TN, Ramsingh G, Young AL, et al. The role of TP53 mutations in the origin and evolution of therapy-related AML. Nature 2015;518:552-555.

22. Donaldson J, Park BH. Circulating tumor DNA: measurement and clinical utility. Annu Rev Med 2018;69:223-234.

23. Grati FR, Malvestiti F, Ferreira JC, et al. Fetoplacental mosaicism: potential implications for false-positive and false-negative noninvasive prenatal screening results. Genet Med 2014;16:620-624.

24. Chan $\mathrm{KC}$, Zhang J, Hui AB, et al. Size distributions of maternal and fetal DNA in maternal plasma. Clin Chem 2004;50:88-92.

25. Flori E, Doray B, Gautier E, et al. Circulating cell-free fetal DNA in maternal serum appears to originate from cyto- and syncytio-trophoblastic cells. Case report. Hum Reprod 2004;19: 723-724.

26. Lo YM, Zhang J, Leung TN, et al. Rapid clearance of fetal DNA from maternal plasma. Am J Hum Genet 1999;64:218-224.

27. Coffee B, Mancini-DiNardo D, Cox HC, et al. A significant proportion of TP53 pathogenic variants detected with a hereditary pan-cancer NGS panel are somatically acquired [abstract]. Presented at 2017 Annual Clinical Genetics Meeting; March 22-24, 2017; Phoenix, Arizona. 